results from the study for monitoring antimicrobial resistance trends (SMART) program: 2009-2011. Clin Ther 2013;35:872-877.

7. Atalay HA, Canat L, Alkan I, Cakir SS, Altunrende F. Prostate-specific antigen reduction after empiric antibiotic treatment does not rule out biopsy in patients with lower urinary tract symptoms: prospective, controlled, singlecenter study. Prostate Int 2017;5:59-64.

8. Eggener SE, Large MC, Gerber GS, et al. Empiric antibiotics for an elevated prostate-specific antigen (PSA) level: a randomised, prospective, controlled multi-institutional trial. BJU Int 2013;112:925-929.

\title{
Surveillance of hospital water systems to help predict the risk of nosocomial legionellosis-Consider other factors
}

\author{
Hilary Humphreys MD, DSc ${ }^{1,2}$, Peter O'Donovan MBS ${ }^{3}$ and Sharon Dwyer $\mathrm{MSc}^{4}$ \\ ${ }^{1}$ Department of Clinical Microbiology, the Royal College of Surgeons in Ireland, Dublin, Ireland, ${ }^{2}$ Department of Microbiology, Beaumont Hospital, Dublin, Ireland, \\ ${ }^{3}$ Department of Estates and Engineering and Facility Management, Beaumont Hospital, Dublin, Ireland and ${ }^{4}$ Department of Quality and Patient Safety, \\ Beaumont Hospital, Dublin, Ireland
}

To the Editor-We read with interest the study by Nagy et $\mathrm{al}^{1}$ in which they used a statistical fit test to support recommendations on the proportion of positive Legionella tests to indicate a wellmaintained or a well-treated water distribution system. They concluded that surveillance showing a rate of $\leq 30 \%$ water samples positive for Legionella spp provided some reassurance. However, some consideration needs to be given to the healthcare facility, the proportion of at-risk patients and the laboratory diagnosis of clinical cases. ${ }^{2}$ Furthermore, there may be a role for considering how heavy the bioburden is in positive water outlet samples and even possibly the Legionella spp.

Beaumont Hospital is a tertiary-care referral center in Ireland with national specialties in renal transplantation, neurosurgery, cochlear implantation, and several other complex specialties. We have an ongoing multidisciplinary team that oversees and delivers a legionellosis prevention program that includes surveillance and the maintenance of a complex hospital facility that is $>30$ years old. This surveillance includes a range of engineering controls (eg, active review of pipework and removal of dead legs); barcoding of assets; active cloud-based temperature checks on the water system; a comprehensive flushing program; and a blended approach to chemical water treatment with chlorine dioxide, copper silver, and anolyte (a positively charged form of chlorine). Our water outlet sampling regimen includes taking 160 samples per month and following up on any positive outlet to optimize operational conditions with retesting until negative. We treat individual water outlets even if the counts are low and whatever the Legionella spp because of the increasingly complex case mix in our hospital. In recent years, we have seen the proportion of water samples positive for any Legionella spp decline from $>30 \%$ to $<2.5 \%$.

European guidelines are very general in terms of sampling; they recommend "periodic sampling and testing for the presence of Legionella based on risk assessment." 3 They further recommend that the sensitivity of laboratory methods should be capable

\footnotetext{
Author for correspondence: Hilary Humphreys, E-mail: hhumphreys@rcsi.ie

Cite this article: Humphreys H, O’Donovan P, and Dwyer S. (2022). Surveillance of hospital water systems to help predict the risk of nosocomial legionellosis-Consider other factors. Infection Control \& Hospital Epidemiology, 43: 1080-1081, https:// doi.org/10.1017/ice.2021.173
}

of detecting counts of $100 \mathrm{CFU} / \mathrm{L}$ or less but that the results should be appropriately interpreted. ${ }^{3}$ Irish guidelines are more specific and include recommendations on sample types, laboratory analysis and action to be taken if an outlet is positive (eg, if $>1,000 \mathrm{CFU} / \mathrm{L}$ ), control measures, and disinfection of the positive water plus resampling. ${ }^{4}$ However, they do emphasize that "Sampling is not a substitute for good maintenance practices and water treatment." 4

Stout et $\mathrm{al}^{5}$ originally identified the correlation between cases of nosocomial legionellosis and $\geq 30 \%$ of outlets being positive. It seems plausible to assume that the more outlets that are positive, the greater the likelihood of nosocomial legionellosis. However, like most infections, healthcare-acquired legionellosis is multifactorial and will be influenced by the vulnerability or susceptibility of the patient (eg, underlying immunosuppression), how the patient is exposed to water (ie, via aerosols), potentially how heavy the bioburden is in the water outlet, and the species. Although we have traditionally regarded Legionella pneumophilia (especially serogroup 1) as the most pathogenic, other serogroups and species may potentially infect susceptible patients, and some general medical and surgical patients are increasingly on biological agents (eg, for inflammatory bowel disease).

Although we do not disagree with the suggestions and recommendations from Nagy et al, we urge caution in overly relying on the proportion of water outlets positive for Legionella as indicating a relatively lower risk of legionellosis. However, further studies are required to determine the influence of case mix, the number and frequency of surveillance samples taken, where they are taken (eg, the clinical area and water outlet such as shower versus hand-wash basin), existing and ongoing preventative measures, and both the bioburden and Legionella species.

Acknowledgments. We acknowledge the support and input of staff from many disciplines and departments who help prevent nosocomial legionellosis and other infections.

Financial support. The collation of material and the preparation of this manuscript was not supported by external funding but was undertaken during routine clinical and academic activities.

Conflicts of interest. H.H. has received research monies and a consultancy fee from Astellas (Ireland) and Pfizer (Ireland) during the last 4 years but not in areas related to the contents of this manuscript. None of the other authors has a conflict of interest to declare. 


\section{References}

1. Nagy DJ, Dziewulski DM, Codru N, Lauper UL. Understanding the distribution of positive Legionella samples in healthcare-premise water systems: using statistical analysis to determine a distribution for Legionella and to support sample size recommendations. Infect Control Hosp Epidemiol 2021;42:63-68.

2. O’Neill E, Humphreys H. Surveillance of hospital water and primary prevention of nosocomial legionellosis: what is the evidence? J Hosp Infect 2005; 59:273-279.

3. European Society of Clinical Microbiology and Infectious Diseases Study Group for Legionella infections. European technical guidelines for the prevention, control and investigation, of infections caused by Legionella species. European Society of Clinical Microbiology and Infectious Diseases website. https:/www.escmid.org/ fileadmin/src/media/PDFs/3Research_Projects/ESGLI/ESGLI_European_
Technical_Guidelines_for_the_Prevention_Control_and_Investigation_ of_Infections_Caused_by_Legionella_species_June_2017.pdf. Published June 2017. Accessed March 31, 2021.

4. Legionnaire's Disease Subcommittee of the Scientific Advisory Committee. National guidelines for the control of legionellosis in Ireland, 2009. Health Service Executive and the Health Protection Surveillance Centre website. https://www.hpsc.ie/a-z/respiratory/legionellosis/guidance/ nationalguidelinesforthecontroloflegionellosisinireland/File,3936,en.pdf. Published 2009. Accessed March 31, 2021.

5. Stout JE, Muder RR, Mietzner S, et al. Role of environmental surveillance in determining the risk of hospital-acquired legionellosis: a national surveillance study with clinical correlations. Infect Control Hosp Epidemiol 2007;28:818-824.

\title{
Interhospital outbreak of Burkholderia cepacia complex ventilator-associated pneumonia (VAP) caused by contaminated mouthwash in coronavirus disease 2019 (COVID-19) patients
}

\author{
Silvia Maria dos Santos Saalfeld MSc ${ }^{1,2}$, Danielle Rosani Shinohara $\mathrm{MSc}^{1}$, Josy Anne Silva MSc ${ }^{2}$, \\ Maria Emilia Avelar Machado MD², Cecilia Saori Mitsugui MSc ${ }^{2}$, Nathalie Kira Tamura MSc${ }^{2}$, \\ Sheila Alexandra Belini Nishiyama $\mathrm{PhD}^{1}$ and Maria Cristina Bronharo Tognim $\mathrm{PhD}^{1}$ (c) \\ ${ }^{1}$ Department of Basic Health Sciences, State University of Maringá, Maringá, Paraná, Brazil, ${ }^{2}$ Maringá University Hospital, State University of Maringá, Maringá, \\ Paraná, Brazil and ${ }^{3}$ Department of Medicine, State University of Maringá, Maringá, Paraná, Brazil
}

To the Editor-In the global coronavirus disease 2019 (COVID-19) pandemic, up to $80 \%$ of the patients in intensive-care units (ICUs) have required invasive mechanical ventilation (IMV). ${ }^{1}$ Inpatients receiving endotracheal intubation and IMV have increased risk of ventilator-associated pneumonia (VAP).$^{1,2}$

Oral hygiene with chlorhexidine-based mouthwash is an important prevention measure for $\mathrm{VAP}^{3}$; however, outbreaks of Burkholderia cepacia complex associated with these products have been reported. ${ }^{4,5}$ To our knowledge, this is the first report of a VAP outbreak caused by B. cepacia complex in COVID-19 patients admitted in ICUs involving 2 hospitals.

In November and December 2020, in a tertiary-care university hospital (hospital 1) in southern Brazil, 7 patients in a COVID-19 ICU and 3 patients in an adult ICU had positive cultures for $B$. cepacia complex $\left(>10^{6} \mathrm{CFU} / \mathrm{mL}\right)$ from endotracheal aspirate (ETA). During this period, 6 other patients in a mixed ICU in a private hospital (hospital 2) in the same region showed B. cepacia complex-positive cultures (Fig. 1).

As part of the intervention, contact-isolation precautions were implemented for all patients with B. cepacia complex-positive cultures. Microbiological data were reviewed to track the source of this contamination, and as reported previously, hospital 1 had experienced consecutive outbreaks of $B$. cepacia complex as a result of the use of intrinsically contaminated mouthwash, so this source was investigated first. ${ }^{6}$

Author for correspondence: Maria Cristina Bronharo Tognim, E-mail: mcbtognim@ uem.br

Cite this article: Saalfeld SMS, et al. (2022). Interhospital outbreak of Burkholderia cepacia complex ventilator-associated pneumonia (VAP) caused by contaminated mouthwash in coronavirus disease 2019 (COVID-19) patients. Infection Control \& Hospital Epidemiology, 43: 1081-1083, https://doi.org/10.1017/ice.2021.183
Burkholderia cepacia complex isolates recovered from ETA and mouthwashes at hospital 1 were characterized phenotypically using the BD-Phoenix automated system (Becton-Dickinson, Franklin Lakes, NJ). Hospital 2 used the matrix-assisted laser desorption/ionization time-of-flight mass spectrometry (MALDI-TOF MS) system (Bruker Daltonics GmbH, Leipzig, Germany). All isolates (hospitals 1 and 2) were typed using the enterobacterial repetitive intergenic consensus-PCR (ERIC-PCR) technique. ${ }^{7}$ BioNumerics 6.5 software (Applied Maths, Sint-Martens-Latem, Belgium) was used to analyze band patterns.

In total, 16 patients had B. cepacia complex-positive cultures recovered from ETA; 12 (75\%) these patients were hospitalized with COVID-19 (positive RT-PCR for severe acute respiratory coronavirus virus 2 [SARS-CoV-2]) (Fig. 1). The mean age of these patients was 66 years, and $69 \%$ were male. All patients received IMV from the first day of ICU admission. The median time between the beginning of IMV and the first isolation of B. cepacia complex was 14 days (interquartile range [IQR], 9-16).

Burkholderia cepacia complex was recovered $\left(>2.7 \times 10^{5}\right.$ $\mathrm{CFU} / \mathrm{mL}$ ) in unopened mouthwash bottles containing $0.12 \%$ chlorhexidine used in both hospital 1 (batch C9252, $250 \mathrm{~mL}$ ) and hospital 2 (batch C9275, $1000 \mathrm{~mL}$ ), all from the same company. This company's mouthwashes had been used at hospital 1 since January 2020 without isolation of B. cepacia complex in infections.

All isolates evaluated showed $100 \%$ genetic similarity, characterizing a monoclonal outbreak involving 3 ICUs and 2 hospitals caused by B. cepacia (confirmed by MALDI-TOF MS).

The manufacturer of these contaminated batches was implicated in a previous B. cepacia complex outbreak at hospital 1, 4 years prior (data reported by our research group). ${ }^{6}$ In the 